\title{
Seismic - Geomechanical Integration in the Brazilian Pre-Salt, Santos Basin
}

\author{
T.B. Meneguim ${ }^{1 *}$, R.Q.Velloso ${ }^{2}$, A.M.N. Tartarini ${ }^{1}$, T.M.Yamamoto ${ }^{1}$, L.E.S Queiroz, M.P. Almeida ${ }^{1}$
}

${ }^{1}$ PETROBRAS, ${ }^{2}$ Pontifical Catholic University of Rio de Janeiro

Copyright 2019, SBGf - Sociedade Brasileira de Geofísica

This paper was prepared for presentation during the $16^{\text {th }}$ International Congress of the Brazilian Geophysical Society held in Rio de Janeiro, Brazil, 19-22 August 2019.

Contents of this paper were reviewed by the Technical Committee of the $16^{\text {th }}$ International Congress of the Brazilian Geophysical Society and do not necessarily represent any position of the SBGf, its officers or members. Electronic roproduction or represent any position of the SBGf, its officers or members. Electronic reproduction or storage of any part of this paper for commercial purposes without the written consent
of the Brazilian Geophysical Society is prohibited.

\section{Abstract}

It is estimated that carbonate reservoirs contains more than $60 \%$ of the oil reserves and $40 \%$ of the gas reserves in the world [1].

The carbonate reservoirs of the brazilian offshore pre-salt layer, Santos Basin, are deep reservoirs $(\sim 5000 \mathrm{~m})$ located in ultra-deep water column $(\sim 2200 \mathrm{~m})$ in a large distance $(\sim 300 \mathrm{~km})$ east from Rio de Janeiro city and exhibit high heterogeneity in terms of porosity, permeability and mechanical properties.

The research on seismic - geomechanical integration aims to develop methodology and anticipate the results when using seismic to improve geomechanical modeling and mitigate risks associated

with : subsidence of the seabed and faults/fractures reactivation as a result of the reservoir production throughout the productive life of the area.

One of the main results of this work was estimate the mechanical properties of deformability:

Young's modulus and Poisson's coefficient, poroelasticity: Biot's coefficient and failure criteria: cohesion and internal friction angle, each one integrated to the seismic data. Another important result was dealing with uncertainties involving the dynamical mechanical properties estimated from seismic, in this sense were created scenarios for these mechanical properties using different dynamic vs. static relations and for different loading conditions: drained or undrained.

\section{Introduction}

The existence of supergiant hydrocarbons accumulations in the carbonate reservoirs of the Brazilian offshore PreSalt layer, Santos Basin, indicate their economical and energetical strategical role.

Since they are complex carbonatic deep reservoirs in ultra-deep water column, the development of the production must be allied to a detailed geomechanical characterization of the area.

The multidisciplinarity of geomechanics applied to hydrocarbon reservoirs is evidenced by its connection not only with the classical geotechnical disciplines: rock/soil mechanics and constitutive models, but also with the disciplines: petroleum engineering, geophysics and geology which must be considered in order to better understand the critical issues present during the exploration and production phases: subsidence of the seabed, faults / fractures reactivation, seal integrity, fluid leakage, hydraulic fracturing pressure, well trajectory optimization, $\mathrm{CO} 2$ injection and temperature variation.

In that context, despite being an indirect measure, high definition seismic information allows to add valuable content to geomechanical modeling through a better understanding of the heterogeneous rock in terms of its mechanical properties, as well as the structural characterization of faults and natural corridors of fractures. These discontinuities can reach tens or hundreds of meters width and height and represent preferred conduits for fluids migration.

The understanding of the seismic amplitude as a contrast of mechanical properties between layers and their relation with deformability, compressibility and poroelasticity properties is the key to seismic- geomechanical integration and an important step in the evolution of the geomechanical modeling that aims at a greater realism and accuracy.

Nowadays the available high resolution seismic data viabilize vertical resolution of $\sim 30-40 \mathrm{~m}$ and horizontal of $\sim 600-800 \mathrm{~m}$ in areas with good signal-to-noise ratio, even in deep and complex regions such as the Brazilian Pre-Salt layer

The research focus is to incorporate geologic features and stratigraphy observed in seismic data and wells into a high performance geomechanical modelling.

It is also essential to seismic-geomechanical integration methodology the adequate equivalence involving dynamic mechanical properties estimated from seismic (very low rock strains $\sim 10^{-7}$ and load - unload - reload cycles of tens $\mathrm{Hz}$ order), well logs (very low rock strains with ultrasonic cycles of $\mathrm{MHz}$ order) and more reliable mechanical properties to the reservoir management: static mechanical properties (load-unload-reload cycles of minutes and high rock strain).

In each of the above cases, the different rock deformations scales as well as the distinct frequency range of load-unload-reload cycles, make non-trivial the dynamic-static equivalence problem.

For the Brazilian Pre-Salt layer, Santos Basin, these relations were obtained from mechanical tests carried out on carbonate reservoirs core samples and for the remaining layers: Salt and Pos-Salt, were used scientific 
journals and reference sources relations [2] due to absence of core samples rocks from these sections.

The static, non-drained and integrated with seismic data mechanical properties are well suited to the non-drained geomechanical simulation. However, due to different permoporosity system for each lithology and loading condition throughout productive life of the area, it is not a very well understood phenomenon what should be the exact behavior of subsurface rock: undrained, partially drained or even drained.

Thus, in order to speculate about drained geomechanical simulation, it were also obtained scenarios of static drained mechanical properties using Gassmann Equation to drained to non-drained conversion.

It is also emphasized that the non-purely elastic but rather elastoplastic or even viscoelastoplastic behavior of the subsurface rocks, should be considered in the mechanical properties modeling since Santos Basin rocks exhibit viscosity (Salt section) and some degree of plasticity (Pre-Salt layer).

However only elastic properties and failure criteria capable of support an elastoplastic simulator will be addressed in this work.

The estimative of mechanical properties to feed more complex mechanical simulators is beyond the scope of this work and can be approached in future researches.

\section{Method}

To ensure seismic integration, the initial point to perform an seismic inversion are seismic and well log data available for the area.

The model based seismic inversion process aims to estimate the petrophysical and mechanical properties such as : porosity, lithology, fluid, density and stiffness matrix of the subsurface rock through the seismic reflection data.

Roughly, this type of inversion is based on the convolution of a reflectivity initial model with an estimated wavelet, comparison of the modeled and input seismic data, and then reflectivity model update to minimize the difference between the modeled and acquired seismic traces [3]

The main product obtained is acoustic impedance (Al) volume that will be used to estimate the medium mechanical properties.

When propagating in subsurface, the seismic pulse is affected by the dispersion phenomenon. In this sense, to better estimate the medium properties in the seismic inversion, it is important to considerer the changing in the seismic pulse shape throughout the burial depth (FIG 1)

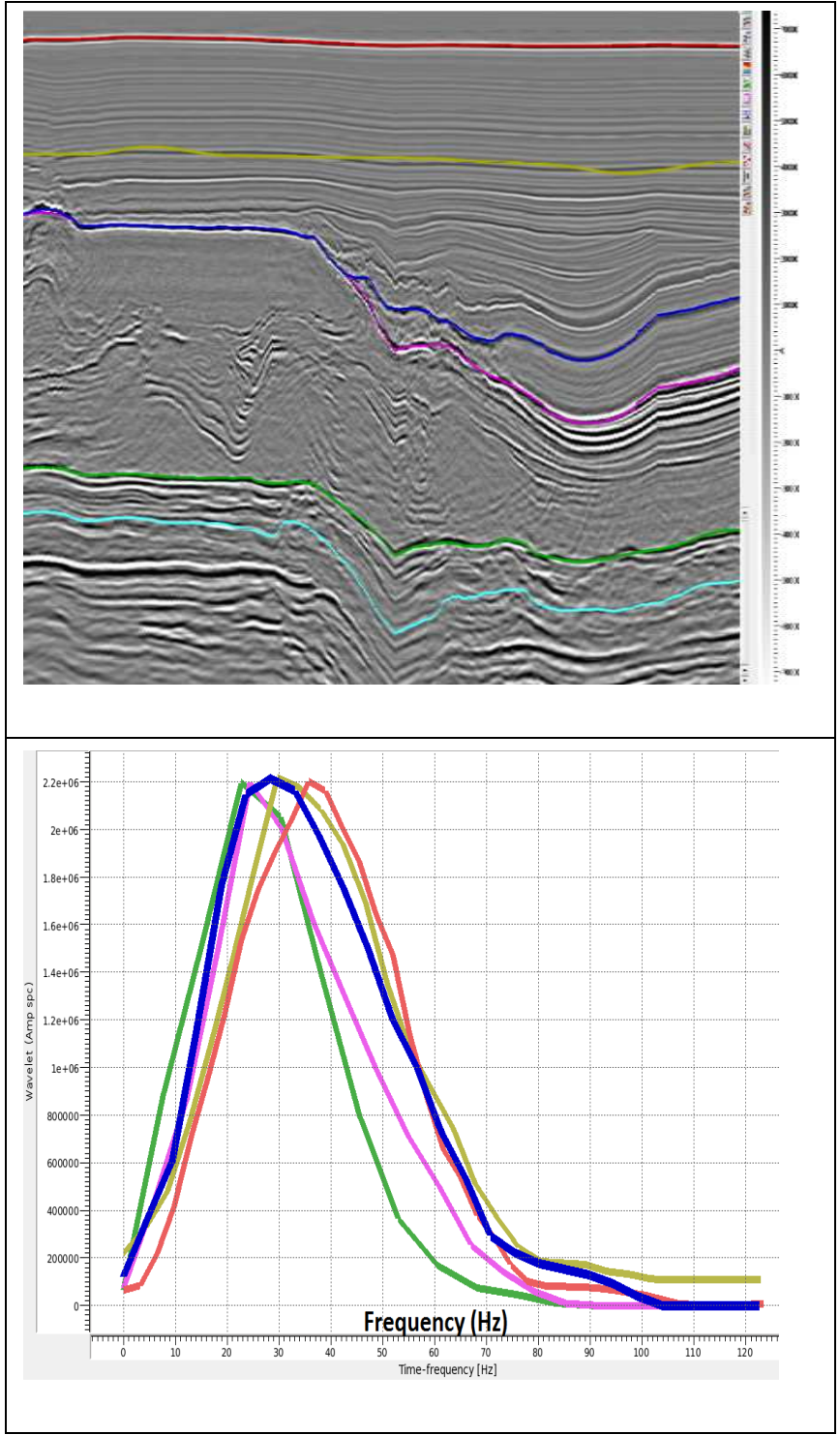

FIG1: (Above) Seismic Session in Migrated Time with Seismic Horizons \& (Below) Seismic Pulse Normalized Amplitude Spectrum throughout subsurface layers

\section{Results}

As the result of seismic inversion using estimated wavelets (FIG 1), seismic 3D data (FIG 2) and well log data available in area, it was estimated mechanical properties (FIG 3) 


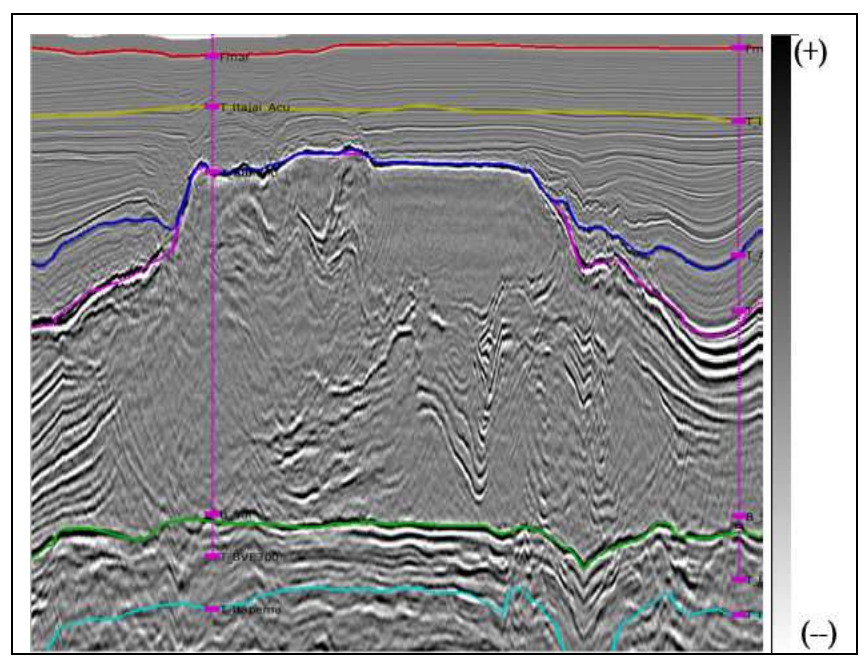

FIG 2: Migrated seismic section - full stack

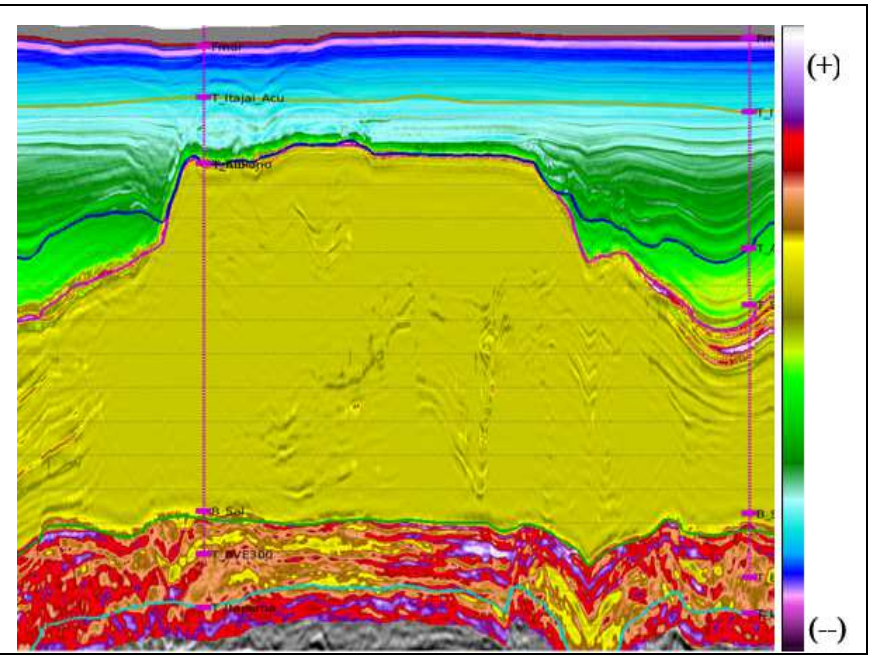

FIG 3: Estimated Dynamic Undrained Young Modulus from Seismic Inversion

Once we've evaluated dynamical undrained mechanical properties from seismic inversion, we should make scenarios involving dynamic vs. static and undrained vs. drained equivalence in order to face uncertainties issues regarding mechanical properties estimative.

By using, Gassmann equation [4] and dynamical vs. static equivalences [2] to: clay, shale, sandstone and marl (PosSalt) and Pre-Salt carbonatic rocks it were computed three scenarios for static undrained Young modulus.

Next, in FIG 4 it is shown for a hypotetic well path the comparison between Dynamic Undrained Young Modulus (black) and three scenarios for Static Undrained Young Modulus ( blue, green and red) throughout stratigraphic section: clay (light green), shale (dark green), sandstone (yellow) and marl (blue) (Pos-Salt) and Pre-Salt carbonatic rocks (cyan) .
Data exhibited in FIG 4 were extracted from our 3D model.

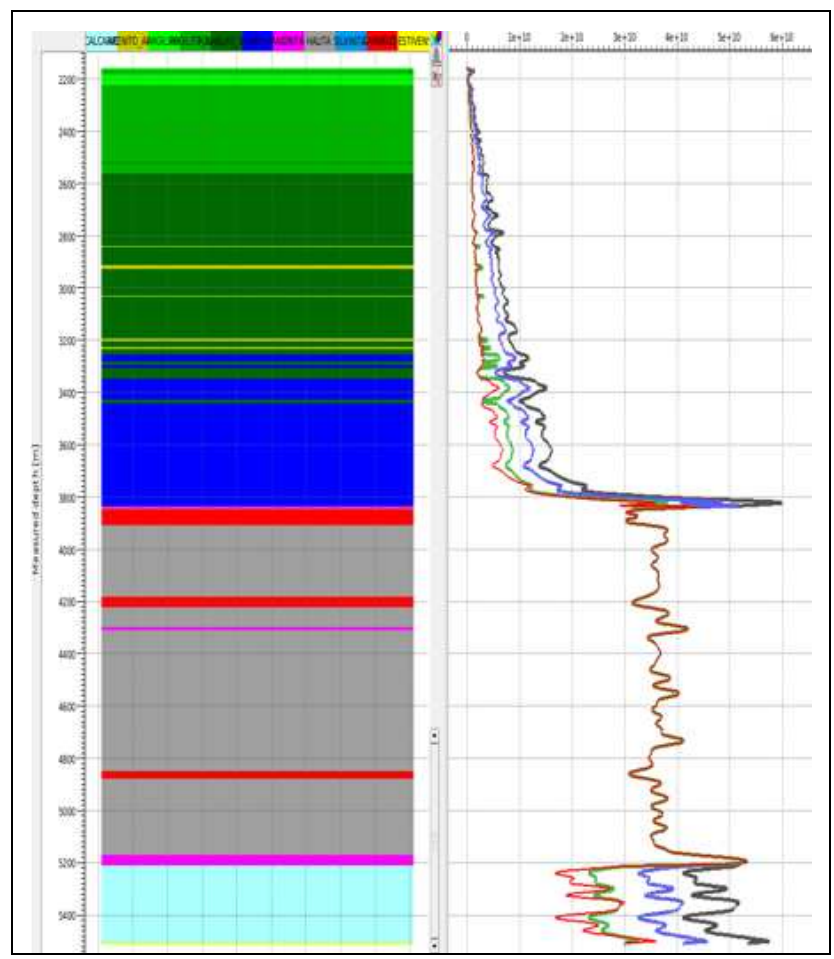

FIG 4: (Right) Three scenarios for Static Undrained Young Modulus (blue, green and red) compared to Dynamic Undrained Young Modulus (black) \& (Left) Lithology Model

\section{Conclusions}

High resolution seismic together well log data must be used to build high detailed and geological geomechanical models.

Estimating dynamical undrained mechanical properties from model based seismic inversion is the key to seismic - geomechanical integration.

Scenarios of static undrained Young modulus can differ up to $30 \%$ depending on static vs. dynamic equivalence and permoporosity system for each lithotype .

\section{Acknowledgments}

The authors would like to thanks Petrobras for allowing the presentation of this work, as well as ANP for the release of seismic data and wells used.

The authors would also like to express their thanks to the following researchers: Guilherme Vasquez and Julio Justen by his contribution in petroacoustic signature of carbonate rock and Erick Slis Raggio by his advices concerning dynamic vs. static issue. 


\section{References}

[1] Schlumberger Carbonate Reservoirs Market Analysis, 2007 and World Energy Outlook, 2006.

[2] Savich A. I. Generalized relations between static and dynamic indices of rock deformability. Translated from Gidrotekhnieheskoe Stroitel'stvo 8, 50-54 (1984).

[3] Li, Q., 2002, Sparse Spike Inversion and the Resolution Limit: CSEG, October 28, 2017

[4] Adam, L., M. Batzle, and I. Brevik, 2006, Gassmann's fluid substitution and shear modulus variability in carbonates at laboratory seismic and ultrasonic frequencies: Geophysics, 71, no. 6, F173-F183.

[5] Ferreira, N.C., Marchesi, V., Gonçalves , C., Fontoura S. , Yao, A. Modeling of Physical and Mechanical Properties, for Drilling Purposes, through 3D Reflection Seismic in the Salt Section : SBMR, 2018 